\title{
PHOTOELECTRIC OBSERVATIONS OF THE OCCULTATIONS OF BINARIES BY THE MOON
}

\author{
K. D. RAKOS \\ University Observatory, Vienna, Austria
}

Recently, I have carried out, at Lowell Observatory in Flagstaff, very extensive observations of occultations of stars by Moon, in order to get the star diameters, the multiple components and the precise time of occultations by analysis of the distortion of diffraction patterns.

The greatest difficulty in attempting to carry out such observations from groundbased facilities lies in the presence of the Earth's atmosphere. Since the Moon's orbit only sweeps out a band of $10^{\circ}$ width in the sky during a period of $18.6 \mathrm{yr}$, it would require a very long time for occultations of significant numbers of different stars to occur for a given location. The number of observable occultations would furthermore be greatly reduced by given weather conditions. One of the greatest disadvantages, however, lies in the fact that one is not in a position to repeat the observations of a given star within a short time. To illustrate these difficulties, I might mention that during the year 1968 occultations of about 500 stars might have been observable at Flagstaff; the atmospheric and weather conditions alone, however, prevented observations of all but 75 stars. It was possible to observe only 5 stars, from about 25 bright stars with the significant apparent diameter.

At that time the idea is born to propose the observations of occultations by means of a Moon based telescope.

\section{Background}

Our aim is to measure the distortion of the diffraction pattern produced by stars of apparent diameters as small as $\alpha=0.001$ arc sec.

From the well-known formulae for diffraction, we have

$$
\begin{aligned}
J(u) & =J_{0} / 2\left\{\left(C(u)+\frac{1}{2}\right)^{2}+\left(S(u)+\frac{1}{2}\right)^{2}\right\}, \\
C(u) & =\int_{0}^{u} \cos \frac{\pi}{2} \cdot \beta^{2} \mathrm{~d} \beta, \quad S(u)=\int_{0}^{u} \sin \frac{\pi}{2} \cdot \beta^{2} \mathrm{~d} \beta, \\
u & =h(2 / b \lambda)^{1 / 2}=\alpha^{\prime \prime} \operatorname{arc} 1^{\prime \prime}(2 b / \lambda)^{1 / 2}, \quad z=\frac{\pi}{2} \cdot u^{2} .
\end{aligned}
$$

(If $J(u)$ has a maximum at $z=z_{1}$ then the next maximum occurs at $z=z_{1}+6$.)

One finds that the intensity of starlight $J(u)$ must be measured to an accuracy of somewhat better than $0.1 \%$, in order that one may in general recognize the difference between $J(u)$ and $J(u)_{\alpha}$ : namely,

$$
J(u)_{\alpha}=J(u) * J(u+\Delta u) \mathrm{d} u,
$$


since

$$
\Delta u=10^{-3} \text { for } \alpha=0.001 \text { arc sec. }
$$

$J(u)$ is the computed or observed value for an infinitely small angular diameter (point source) and $J(u)_{\alpha}$ the observed value of the distorted diffraction pattern of a star with angular diameter $\alpha$.

Using a band width of only $50 \AA$ at about $5000 \AA$, we can expect to recognize about 100 maxima in the diffraction pattern; this corresponds to a value $u=20$.

\section{Telescope}

For the design of the Moon based Telescope I have adopted the following suppositions:

The full aperture of the telescope is $50 \times 50 \mathrm{~cm}$. The mirror consists of 50 cylindrical (or better 10000 spherical) polished sections. These are oriented with respect to one another in such a way that the images of a star under observation are contained in the $2 \times 2 \mathrm{~cm}$ area of a SEC TV camera tube. The minimum resolution of the mirror in the direction of the cylinder axis shall be $0.05 \mathrm{~mm}$. The integrating time of the SEC tube should be about $1 \mathrm{sec}$ and transmission should be in accordance with standard techniques.

\section{Telescope Controls}

In order to find and set up on a star, the telescope is to be movable through 20 deg in horizontal direction. In order to achieve the necessary integration time of $1 \mathrm{sec}$, the mirror shall be movable parallel to itself through about $5 \mathrm{~cm}$ in vertical direction. The accuracy of the horizontal motion should be \pm 1 arc min, the vertical motion should be uniform to $\pm 0.03 \mathrm{~mm}$.

\section{Spectral Regions}

For measurements of stellar diameters and of double stars three different interference filters of at most $50 \AA$ bandwidths should be available for placement in front of the cathode of the SEC tube (Westinghouse WX 30677+WX 30691). For photometry of stars additional photomultipliers and filters should be available, particularly for observations in UV.

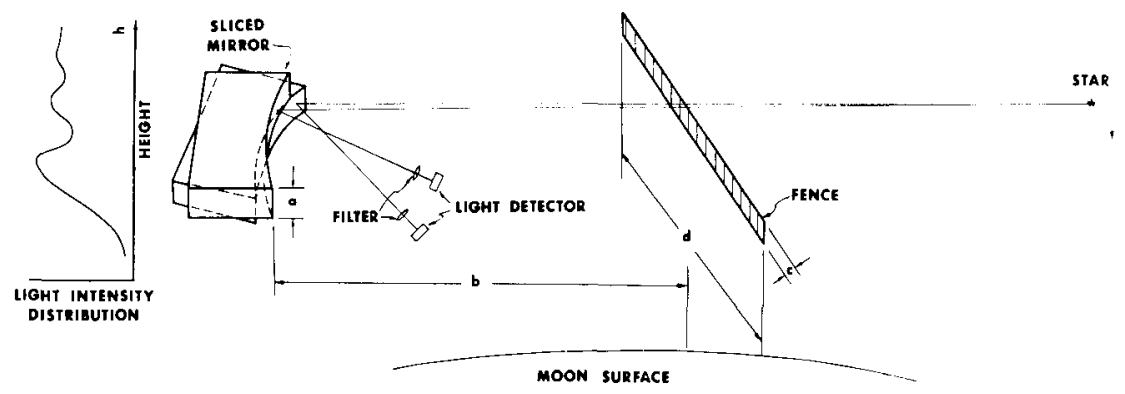

Fig. 1. Measurement of stellar diameters from the Moon's surface. 


\section{The Diffracting Edge}

A band, 1 in. wide, should be placed at $10 \mathrm{~km}$ distance to serve as the diffracting edge (fence). The minimum height above the lunar surface should be $100 \mathrm{~cm}$ and the length should correspond to $20 \mathrm{deg}$ as seen from the telescope. The band can be laid out in sections. It must be parallel with the sections of the mirror to within $\pm 5 \mathrm{deg}$. Even a considerably shorter length of the fence, for example $5 \mathrm{deg}$, instead of $20 \mathrm{deg}$, would not seriously decrease the number of observable stars, since the orbital plane of the moon shifts along the ecliptic with a period of $18.6 \mathrm{yr}$; in this case, however, one would have to operate the instrument on the lunar surface for a much longer time to obtain the same coverage of objects reached with the longer fence.

\section{Accuracy of Measures}

Since the SEC tube generally has a resolution of 20 line pairs per millimeter, it will not be difficult to measure the function $J(u)_{\alpha}$ in steps of $\Delta u=0.001$ for all values $0<u<20$.

The function $J(u)_{\alpha}$ cannot be recorded entirely at once with the telescope, since in order to do so, the mirror would have to be twice as high as specified earlier.

( $\Delta u=0.001$ corresponds to $0.05 \mathrm{~mm}$ in the direction of the apparent motion of the star or in the direction of the axis of the mirror cylinder.)

Because of the motion of the star, however (maximum $0.5 \mathrm{sec}^{-1}$ ) all values of the function do occur in the observational sequence.

In order to achieve the integration time of $1 \mathrm{sec}$, the mirror must be moved for $1 \mathrm{sec}$ in vertical direction to compensate for the motion of the star relative to the diffracting edge. This rate of motion results in a total vertical movement of about $5 \mathrm{~cm}$. After having been lifted to its extreme position, the mirror should be returned to its starting position as rapidly as possible. During this period read out from SEC tube takes place. Immediately following the simultaneous read out of the tube and return of the mirror to its starting position, the next integration cycle commences. Measurement of the entire diffraction pattern, at one of the 2 edges and to $u=20$, will require slightly more than $20 \mathrm{sec}$. Measurement at both edges of the fence will require about $40 \mathrm{sec}$.

Considering that a star of visual magnitude $V=0$ produces about $2.4 \times 10^{3}$ photons/ $\mathrm{cm} \AA$ sec above the Earth's atmosphere and assuming the quantum efficiency to be roughly $20 \%$, one can easily show that the total number of photoelectrons obtained during measurement of a star of $V=7^{m}$ is larger than $10^{6}$. This number assures the necessary statistical accuracy of $0.1 \%$.

\section{Expected Results}

A check of the Bright Star Catalogue shows that within the band of declination $-10^{\circ}$ to $+10^{\circ}$ there are about 400 stars to $V=6^{m} .5$ (limiting magnitude of the catalogue) whose diameters exceed $0.00 \mathrm{I}$ arc sec. However, since stars of spectral type $M$ down 
to $V=8^{m} .5$ generally have angular diameters larger than 0.002 arc sec, one can practically expect to be able to measure the diameters of about 900 stars. During the course of one year the diameter determination of any given star could be repeated at least five times. Should the equipment remain functional for a longer period of time, then at least 500 more stars could be added to the observing list because of the gradual shift of the plane of the lunar orbit relative to the ecliptic.

Aside from the Sun and certain double stars, there are only very few stars whose diameters have been measured directly. It is well known that calculation of a stellar radius from the stars luminosity and mass by means of the theory of stellar interior is not entirely free of assumptions. Accumulation of a large number of direct measurements of stellar diameters will provide checks on assumptions underlying these theories and will yield improved knowledge concerning the internal structure of stars, the center to limb darkening, the structure of stellar atmospheres, the effective temperatures of stars, the distance scale in space, etc.

The incidence of duplicity among stars and the orbital and physical characteristics of the components of binaries have important bearing on theories of the origin and evolution of stars. Data obtained from Earth-based observations are extensive, but are nevertheless affected by severe limitations. The closest pairs detected and observed systematically are eclipsing binaries, spectroscopic binaries and a small number of objects measured by interferometric techniques. The occurrence of observable eclipses depends on rather stringent geometric conditions, while observations by spectroscopic and interferometric methods are limited primarily by the effective magnitude differences between the components. One may therefore assume that a large percentage of close double stars of separations between 0.1 and 0.001 arc sec and with brightness differences between the components of several magnitudes has remained undetected. The Moon-based instrument will allow pairs of $0.001 \mathrm{arcsec}$ separation to be detected if the components are of equal brightness. Pairs with magnitude difference of $5^{m}$ could be found and measured if their separations were 0.05 arc sec or larger. Since many of these very close pairs can be expected to be binaries of very short periods, repeated observations will in many cases permit measurement and subsequent evaluation of their orbital motions.

Of the 400 bright stars, selected from the Bright Star Catalogue and referred to earlier, 47 are known to be variable. Observations of these stars would provide important checks on the theories concerning these objects, since they would yield accurate photometry and, at the same time, determinations of the diameters of these stars.

It would be possible to establish precise photometric sequences, consisting of several thousand stars, on different photometric systems. The accuracy of these observations would be higher by an order of magnitude than that obtainable from Earth. Furthermore, it would be possible to carry out extremely important measures of the far ultraviolet, a region totally inaccessible to Earth-based observations. It is virtually impossible to judge what effect such observations might have on our present knowledge of the astronomical universe. 


\section{References}

Rakos, Karl D.: 1967, 'Die Bestimmung von Sterndurchmessern mit Hilfe der Verzerrung der Fresnelschen Beugungsfigur bei der Bedeckung durch den Mond', Teil I, Acta Physica Austriaca 26, 152-172; Teil II, 26, 289-307.

\section{Discussion}

Heintz: How are the chances of separating diameters and limb darkening coefficients by using widely different wavelength bands?

Rakos: This is of course possible, you need at least two different wavelengths. My suggestion is to observe the occultation in the same time using two different filters and a blue and a red photomultiplier. The information in both channels, blue and red, should be recorded on the magnetic tape.

Fracastoro: Astrophysics on the Moon of course is very promising. In this special case, however, I wonder whether, after landed on the Moon, the screen might be placed as far as $10 \mathrm{~km}$ away from the landing point.

Rakos: Dr W. Hess, scientific director in NASA, told me, the $10 \mathrm{~km}$ distance from the landing site is within the range of the future Moon excursions. 